\title{
Produção brasileira em periódicos psiquiátricos de alto fator de impacto em 2005
}

\author{
Brazilian production in psychiatric journals with high impact factor in 2005
}

\author{
Dante Galileu, Felipe Filardi da Rocha, Rodrigo Nicolato, Antonio Lucio Teixeira, Marco \\ Aurélio Romano-Silva e Humberto Correa
}

\begin{abstract}
Resumo
Objetivo: Avaliar a produção científica brasileira, no ano de 2005, nas 20 revistas de psiquiatria com maior fator de impacto $(\mathrm{FI})$ segundo o Journal of Citation Report (JCR), 2004. Métodos: Do total de 90 periódicos com FI variando de 11,207 até 0,13 , selecionamos todos os artigos dos periódicos com Fl acima do percentil $75(n=20)$. Avaliamos, entre esses artigos, quantos apresentavam pelo menos um autor brasileiro, quantos eram compostos por equipe exclusivamente brasileira ou tinham pelo menos o primeiro autor e/ou 0 autor sênior filiado à instituição nacional. Avaliamos também a respectiva distribuição das publicações por estados de acordo com a instituição de filiação do(s) autor(es). Resultados: Entre 4.859 artigos avaliados, $54(1,11 \%)$ tinham pelo menos um autor com afiliação a instituição brasileira e 22 tinham autor sênior brasileiro, excluindo-se as 32 publicações compostas exclusivamente por pesquisadores brasileiros. Ao se determinar a distribuição, por estados, da instituição de pesquisa à qual está(ão) vinculado(s) o(s) autor(res) brasileiro(s), São Paulo, com 34 publicações $(55,73 \%)$, ficou na primeira posição, com predominância absoluta do eixo Sudeste-Sul (96,72\%). Conclusão: Apesar de as publicações brasileiras em psiquiatria terem apresentado aparente crescimento quantitativo e qualitativo, a produção cientifica da área é ainda pequena e altamente concentrada em alguns poucos estados.

Palavras-chave: análise bibliométrica, periódicos, psiquiatria, pesquisa.
\end{abstract}

\begin{abstract}
Objective: We evaluated the Brazilian scientific production published in the year 2005 in the twenty psychiatric periodicals with high impact factor (IF) according to Journal of Citation Report (JCR), 2004. Methods: From 90 periodicals with IF ranging from 11,207 to 0,13 , we selected all articles from periodicals with IF higher than percentile $75(n=20)$. We assessed which articles had at least one author affiliated to a Brazilian institution as well as articles that had only Brazilian authors or first/senior author belonging to a national institution. Secondly, the state distribution of the author(s) affiliation was determined. Results: Among 4,859 articles examined, $54(1,11 \%)$ had at least one author from a Brazilian institution and 22 had first senior author belonging to a national institution, excluding the 32 publications composed only by Brazilian authors. The distribution by state showed São Paulo at first with 34 articles $(55.73 \%)$ and absolute predominance of South-Southeast region (96.72\%). Conclusion: Although psychiatry Brazilian publications had demonstrated apparent quantitative and qualitatite growing, our scientific production on the area is highly concentrated in a few states.

Key words: bibliometric analysis, periodicals, psychiatry, research.
\end{abstract}

Faculdade de Medicina da Universidade Federal de Minas Gerais (UFMG) e Fundação de Amparo à Pesquisa do Estado de Minas Gerais (FAPEMIG) (Galileu D) Hospital das Clínicas (HC) e Instituto de Ciências Biológicas (ICB) da UFMG (Rocha FF) Universidade José do Rosário Vellano (UNIFENAS); União Educacional do Vale do Aço (UNIVAÇO); Instituto Raul Soares da Fundação Hospitalar do Estado de Minas Gerais (IRS/FHEMIG) e Instituto de Previdência dos Servidores do Estado de Minas Gerais (IPSEMG); ICB/UFMG (Nicolato R)

30-06-06 Faculdade de Medicina da UFMG (Teixeira AL, Correa H) ICB/UFMG (Romano-Silva MA) Trabalho desenvolvido no Serviço de Psiquiatria do HC/UFMG e na Faculdade de Medicina da UFMG. 


\section{Introdução}

A abrangência da circulação de uma publicação (local, nacional ou internacional) transformou-se num parâmetro indicativo de qualidade a partir do final do século XIX. Diante da crescente quantidade de artigos publicados em todo 0 mundo sobre um determinado assunto e da impossibilidade de acompanhar esse crescimento, pesquisadores começaram a desenvolver sistemas que racionalizassem e facilitassem o acesso a essas informações. Criaram então os primeiros abstracts, sistemas de indexação da literatura que, hoje, aliados aos recursos informacionais, apresentam-se como poderosas ferramentas de disseminação da informação científica. Ao longo do tempo, numa escala global, a inclusão de um título de periódico em alguns desses sistemas passou a ser vista como indicativo de prestígio à publicação (Miranda et al., 1996).

Em 1958 foi criado na Filadélfia, Estados Unidos, o Institute for Scientific Information (ISI), que passou a avaliar a produção científica mundial nas mais diversas áreas. Para que um periódico ingresse nessa base de dados ele deve reunir requisitos como pontualidade de publicação e cumprimento das normas internacionais de editoração (título informativo, correção das referências citadas, informações completas dos autores, pelo menos título, resumo e descritores em inglês, e processo de revisão dos artigos a serem publicados por pares - peer review). 0 critério de seleção é rigoroso, uma vez que o Institute for Scientific Information (ISI) tem o compromisso de oferecer a cobertura completa dos periódicos mais importantes e influentes do mundo para atualização de informações e entende que tal cobertura deva ser feita não pela quantidade, mas pela qualidade (Garfield, 1995).

O ISI avalia o desempenho de cerca de 3.500 periódicos indexados através do Journal of Citation Reports (JCR). O principal parâmetro utilizado é o chamado fator de impacto (FI), o qual é calculado pela divisão do número de citações que um periódico recebe ao longo de dois anos pelo número de todos os artigos nele publicados nesse mesmo período. Assim, quanto maior tiver sido o FI de um periódico, mais vezes, na média, seus artigos foram citados em outros periódicos, o que nos dá uma medida, pelo menos indireta, da qualidade e da relevância dessas publicações (Garfield, 1995; Moed, 2002)

Em 2005, Bressan, Gerolin e Mari avaliaram a produção científica nacional das áreas de psiquiatria, psicobiologia e saúde mental, no período compreendido entre 1998 e 2002, em nove programas nacionais de pós-graduação stricto sensu das áreas citadas. Esses autores concluem que, segundo dados do JCR de 2004, o Brasil é o 28 país no ranking de citações e número de artigos indexados no ISI na área de psiquiatria/psicologia. Além disso, o Brasil passa por fase de crescimento de publicações de psiquiatria e áreas afins em jornais indexados no ISI, demonstrada, entre outras coisas, pelo fato de entre 1998 e 2002 o número de publicações no ISI ter dobrado (Bressan et al., 2005).

No JCR de 2004 constam 90 periódicos de psiquiatria e seus respectivos Fls. Observamos, entretanto, uma enorme variação entre eles. Entre o Archives of General Psychiatry
$(F I=11,2)$, que se encontra em primeiro lugar no ranking, e o Zh Nevropatol Psik (FI = 0,13), em 90, há uma diferença de 86 vezes no $\mathrm{Fl}$. Ou seja, em média, um artigo publicado na primeira revista é 86 vezes mais citado do que um outro publicado na segunda revista (JCR, 2004).

Tendo em vista essa discrepância, objetivamos no presente estudo avaliar a produção brasileira, no ano de 2005, nas 20 revistas de psiquiatria com maior FI segundo o JCR de 2004, possibilitando perceber a verdadeira contribuição da pesquisa brasileira para os periódicos de maior influência.

\section{Métodos}

Através do site http://esi3.isiknowledge.com/rankdatapage.cgi, acessado em 1 de abril de 2006, obtivemos o JCR de 2004, onde constam 90 periódicos de psiquiatria com FI variando de 11,2 até $0,13(2,2 \pm 1,8)$. Para nosso estudo selecionamos todos os periódicos que se enquadram no percentil 75 (FI $\geq 3.042)$, totalizando 20 periódicos.

Cada periódico tem formato diferente e publica artigos com diversas características, como editoriais, revisões, cartas, artigos completos, artigos breves, comentários, entre outros. Consideramos em nossa análise todos os tipos de artigo, excetuando-se apenas comentários sobre livros e erratas.

Entre esses artigos avaliamos inicialmente aqueles em que pelo menos um autor tem afiliação a instituição brasileira. A seguir analisamos os artigos em que pelo menos o primeiro autor e/ou o autor sênior têm afiliação a instituição brasileira e artigos em que todos os autores apresentam afiliação a instituição brasileira, ou seja, artigos produzidos por uma equipe trabalhando exclusivamente no Brasil.

A seguir avaliamos, entre os artigos selecionados, a respectiva distribuição das publicações por estados de acordo com a instituição de pesquisa à qual o pesquisador está afiliado.

A análise estatística foi realizada com o programa SPSS v. 14 para Windows.

\section{Resultados}

Os 20 periódicos de psiquiatria indexados no ISI e citados no JCR de 2004 com maior FI $(4,25 \pm 1,97)$ estão listados na Tabela 1.

Para cada periódico obtivemos o número de artigos publicados em 2005 e, a seguir, avaliamos quantos desses artigos, para cada revista, tinham pelo menos um autor com afiliação numa instituição brasileira. No total obtivemos 4.859 artigos, sendo que em 54 deles $(1,11 \%)$ havia pelo menos um autor com afiliação a instituição brasileira. Ao se analisarem os artigos que apresentam autor sênior brasileiro, excluindose aqueles compostos exclusivamente por pesquisadores brasileiros, obtiveram-se seis publicações num total de 22 artigos. Artigos produzidos por equipe exclusivamente brasileira representaram 32 publicações, mais da metade dos artigos publicados com pelo menos um autor brasileiro $(59,25 \%)$ (Tabela 1$)$.

Ao se analisarem os 54 artigos e determinar a distribuição por estados da instituição de pesquisa à qual está(ão) 
Tabela 1. Periódicos de psiquiatria com alto fator de impacto e participação brasileira

\begin{tabular}{|c|c|c|c|c|c|}
\hline Periódico & FI & $\begin{array}{l}\text { Número de } \\
\text { artigos }\end{array}$ & $\begin{array}{l}\text { Número de artigos } \\
\text { com pelo menos um } \\
\text { autor brasileiro }\end{array}$ & $\begin{array}{l}\text { Número de artigos } \\
\text { com autor sênior } \\
\text { brasileiro* }\end{array}$ & $\begin{array}{l}\text { Número de artigos com } \\
\text { autores exclusivamente } \\
\text { brasileiros }\end{array}$ \\
\hline 1. Archives of General Psychiatry & 11,207 & 151 & Nenhum & Nenhum & Nenhum \\
\hline 2. American Journal of Psychiatry & 7,614 & 646 & 9 & Nenhum & 2 \\
\hline 3. Molecular Psychiatry & 6,943 & 143 & 3 & 2 & Nenhum \\
\hline 4. Biological Psychiatry & 6,159 & 476 & 5 & 1 & 2 \\
\hline 5. Neuropsychopharmacology & 4,941 & 246 & 3 & 1 & 1 \\
\hline 6. Sleep & 4,94 & 185 & 2 & Nenhum & 2 \\
\hline 7. The Journal of Clinical Psychiatry & 4,806 & 252 & 2 & Nenhum & 2 \\
\hline $\begin{array}{l}\text { 8. The Journal of Clinical } \\
\text { Psychopharmacology }\end{array}$ & 4,419 & 161 & 1 & Nenhum & 1 \\
\hline 9. British Journal of Psychiatry & 4,175 & 378 & 3 & Nenhum & Nenhum \\
\hline $\begin{array}{l}\text { 10. International Journal of } \\
\text { Neuropsychopharmacology }\end{array}$ & 4,128 & 74 & 4 & Nenhum & 4 \\
\hline 11. Bipolar Disorder & 4,065 & 85 & 2 & Nenhum & Nenhum \\
\hline $\begin{array}{l}\text { 12. Psychotherapy and } \\
\text { Psychosomatics }\end{array}$ & 3,987 & 53 & Nenhum & Nenhum & Nenhum \\
\hline 13. Schizophrenia Research & 3,889 & 325 & 6 & 1 & 5 \\
\hline $\begin{array}{l}\text { 14. European } \\
\text { Neuropsychopharmacology }\end{array}$ & 3,545 & 79 & 2 & Nenhum & 2 \\
\hline $\begin{array}{l}\text { 15. Journal of the American Academy of } \\
\text { Child and Adolescent Psychiatry }\end{array}$ & 3,529 & 318 & 2 & Nenhum & 2 \\
\hline $\begin{array}{l}\text { 16. American Journal of Geriatric } \\
\text { Psychiatry }\end{array}$ & 3,469 & 152 & Nenhum & Nenhum & Nenhum \\
\hline 17. Psychosomatic Medicine & 3,429 & 145 & Nenhum & Nenhum & Nenhum \\
\hline $\begin{array}{l}\text { 18. Psychopharmacology } \\
\text { 19. Mental Retardation and }\end{array}$ & 3,146 & 449 & 10 & 1 & 9 \\
\hline $\begin{array}{l}\text { Developmental Disabilities } \\
\text { Research Reviews }\end{array}$ & 3,114 & 44 & Nenhum & Nenhum & Nenhum \\
\hline $\begin{array}{l}\text { 20. Journal of Neurology, } \\
\text { Neurosurgery and Psychiatry }\end{array}$ & 3,11 & 497 & Nenhum & Nenhum & Nenhum \\
\hline Total & & 4.859 & 54 & 6 & 32 \\
\hline
\end{tabular}

*Excluindo-se os artigos produzidos por equipe composta exclusivamente por autores filiados a instituição brasileira.

vinculado(s) o(s) autor(res) brasileiro(s), obteve-se um total de 61 citações, já que instituições de estados diferentes, em alguns casos, estiveram presentes na mesma publicação devido ao trabalho conjunto entre várias delas. 0 estado de
São Paulo, com 34 publicações $(55,73 \%)$, ficou na primeira posição, seguido pelo Rio Grande do Sul com dez publicações (16,39\%). Há predominância absoluta do eixo Sudeste-Sul, com $96,72 \%$ dos artigos (Tabela 2)

Tabela 2. Distribuição por estado dos artigos publicados nos periódicos de psiquiatria e dos cursos de pós-graduação stricto sensu nas áreas de psiquiatria, saúde mental, neuropsiquiatria e psicobiologia

\begin{tabular}{lcc}
\hline Estados & Número de publicações & $\begin{array}{l}\text { Número de programas de pós-graduação stricto sensu } \\
\text { nas áreas de psiquiatria, saúde mental, } \\
\text { neuropsiquiatria e psicobiologia }\end{array}$ \\
\hline São Paulo & 34 & 5 \\
Rio Grande do Sul & 10 & 1 \\
Rio de Janeiro & 5 & 1 \\
Santa Catarina & 5 & 0 \\
Minas Gerais & 3 & 0 \\
Paraná & 1 & 0 \\
Distrito Federal & 1 & 0 \\
Bahia & 1 & 0 \\
Espírito Santo & 1 & 0 \\
Total & 61 & 7 \\
\hline
\end{tabular}




\section{Discussão}

A produção científica brasileira medida por número de artigos em periódicos científicos internacionais indexados pelo ISI mais que quintuplicou de 1981 a 2002, ocupando, em outubro de 2002, o 22ㅇ lugar no número de publicações, o 25은 em relação à quantidade de citações e o 920 lugar no quesito citações/publicação (In-Cities, 2004). Conseqüentemente houve crescimento das publicações brasileiras de $0,44 \%$ da produção científica mundial, em 1981, para 1,53\%, entre 2000 e 2004. Nesse mesmo período, o Brasil foi responsável por $0,41 \%$ dos artigos publicados em periódicos ligados à pesquisa da área psiquiatria/psicologia, com 3,01 citações/artigo, o melhor índice ao se compararem as inúmeras áreas científicas, apenas $12 \%$ abaixo do índice mundial de 3,43 citações/artigo (In-Cities, 2005). Em nosso estudo, 1,11\% dos artigos publicados nas 20 revistas de maior $\mathrm{Fl}$ apresentam pelo menos um autor brasileiro, enquanto quase $60 \%$ dos artigos são exclusivamente compostos por membros de instituições nacionais. Como nenhum dos 90 periódicos de psiquiatria do JCR de 2004 foi avaliado, não podemos afirmar que houve crescimento do número de artigos com autores brasileiros, mas observa-se, entre os periódicos psiquiátricos de maior relevância, uma importante participação do Brasil. Devemos ainda lembrar que matérias das áreas de psiquiatria, psicobiologia e saúde mental podem ser publicadas em revistas de outras áreas que não a psiquiatria, como genética, neurociência e farmacologia, que não foram avaliadas aqui.

Merece destaque o fato de que, dos 22 artigos em que houve contribuição de brasileiros, $72,72 \%$ tinham o pesquisador nacional como co-autor. O Comitê Internacional de Editores de Revistas Médicas (ICMJE) define que a co-autoria também é autoria (autorship) e que todos os autores devem assumir responsabilidade pública pelo conteúdo publicado. Embora até hoje trabalhos mostrem que é difícil definir com clareza a natureza e o papel da contribuição de co-autores e publicações com diversos autores, o comitê afirma que a ordem de autoria deve ser uma decisão tomada coletivamente. Do ponto de vista da importância, geralmente são considerados principais contribuintes os três primeiros e o último, geralmente o mentor do grupo. Os autores que se situam entre esses extremos são menos importantes. No entanto, quando um autor é citado, os co-autores acabam sendo também automaticamente citados, pois a citação refere-se à obra produzida pelo conjunto dos autores (Almeida, 1998; Shapiro et al., 1994).

Em nosso estudo observamos predominância absoluta dos estados das regiões Sudeste e Sul como origem das instituições a que são afiliados os autores. Já é sabido que a produção científica na área médica está restrita fundamentalmente às grandes escolas, localizadas nessas regiões, e compreende trabalhos não só médicos, mas também de áreas básicas tradicionalmente ligadas à medicina. Independentemente do índice estudado, o eixo Sudeste-Sul é, na maioria dos casos, o mais privilegiado, onde estão os centros de excelência, o maior número de pesquisadores e de cursos de pós-graduação, as grandes editoras e empresas e os meios de comunicação mais poderosos, além, é claro, de ser o pólo da economia nacional (Miranda et al., 1996). Palavras textuais de Castro ditas em
1986 se encontram em plena ressonância com o panorama atual: "a polarização da ciência nos locais mais prósperos...", porque "as regiões Norte, Nordeste e Centro-Oeste (excluindo Brasília) produzem apenas $9,5 \%$ da ciência brasileira, o que equivale a dizer que quase toda a atividade científica está concentrada no Centro-Sul" (Castro, 1986).

Dentro dessa concentração da produção científica nas regiões Sudeste e Sul, observam-se mais desigualdades. Segundo o último censo do Conselho Nacional de Desenvolvimento Científico e Tecnológico (CNPq) realizado em 2004, o Sudeste e o Sul apresentam, respectivamente, $52 \%$ e $21 \%$ das instituições de pesquisa do país (Fundação Coordenação de Aperfeiçoamento de Pessoal de Nível Superior [CAPES], 2004). Entre os nove programas de pós-graduação (PG) das áreas de psiquiatria, psicobiologia e saúde mental existentes no Brasil (ciências médicas: psiquiatria - Universidade Federal do Rio Grande do Sul [UFRGS]; medicina: saúde mental - Universidade de São Paulo/Ribeirão Preto [USP/RP]; neuropsiquiatria e ciências do comportamento - Universidade Federal de Pernambuco [UFPE]; psicobiologia - Universidade Federal do Rio Grande do Norte [UFRN]; psicobiologia - USP/RP; psicobiologia - Universidade Federal de São Paulo [UNIFESP]; psiquiatria - USP; psiquiatria e psicologia médica - UNIFESP; psiquiatria, psicanálise e saúde mental - Universidade Federal do Rio de Janeiro [UFRJ]), seis $(62,5 \%)$ se encontram na região Sudeste, estando cinco no estado de São Paulo e um no Rio de Janeiro.

Os dados obtidos em nosso trabalho apontam para a predominância do número de artigos nos estados que mantêm PG nas áreas citadas (Bressan et al., 2005). Embora não seja 0 foco desse estudo, não deixa de ser sugestiva essa correlação entre a existência dos programas e a produção, o que pode indicar a importância e o vigor do sistema como catalisador da produção científica, qualificando um número cada vez maior de mestres e doutores, pessoas capacitadas a desenvolver e coordenar projetos de pesquisa. Deve-se ressaltar que não há correlação direta entre essas áreas de pesquisa da $P G$ estudadas e os periódicos de psiquiatria avaliados, já que outras áreas, como, por exemplo, a neurociência e a neurologia, podem desenvolver trabalhos correlatos com o perfil de publicação dos periódicos.

É sabido que há uma intrínseca relação entre o ISI e o Index Medicus. Geralmente, periódicos indexados no ISI também o são no sistema norte-americano Index Medicus (National Library of Medicine, Washington), cuja versão informatizada se chama Medline. Contudo muitos jornais indexados no Medline não possuem cálculo de FI. Por exemplo, no Brasil, a Revista Brasileira de Psiquiatria, de qualidade científica indiscutível, é indexada no Medline, mas ainda não possui FI, embora, recentemente, em 2005, tenha sido indexada no ISI e, em breve, deva ter o seu FI divulgado (Bressan et al., 2005). Portanto, ao se tentar estudar a atual participação da pesquisa nacional tanto na área psiquiátrica como em outras, deve-se entender que o ISI não é o único destino dos resultados alcançados. Nossa ciência pode ser representada por um iceberg que tem uma parte visível acima da água, que estaria representando a produção científica brasileira nas bases de dados internacionais, no caso do ISI, e que corresponde a aproximadamente 
$20 \%$ a $25 \%$ do total (Miranda et al., 1996). A produção nacional que não está indexada no ISI corresponde a cerca de $80 \%$ e se mantém submersa, pouco visível. Isso de imediato traz uma preocupação óbvia para todos nós, pesquisadores brasileiros: a de não podermos saber qual é a qualidade dessa produção, qual o impacto de sua circulação e a não-acessibilidade dessa produção por leitores internacionais (Bressan et al., 2005; Shapiro et al., 1994).

\section{Conclusão}

Para resumir, verificamos que, apesar de as publicações brasileiras em psiquiatria terem apresentado aparente crescimento quantitativo e qualitativo, a produção científica da área é ainda insuficiente, principalmente se levarmos em conta a alta prevalência dos transtornos mentais no Brasil, o ônus de sofrimento e o custo a que estão sujeitos os pacientes e a sociedade (Almeida-Filho et al., 1997). Em estudo recente, a Organização Mundial da Saúde (OMS) mostrou que condições neuropsiquiátricas como a depressão unipolar, a dependência do álcool, o transtorno bipolar e a esquizofrenia estão entre as doenças que mais causam "anos vividos com incapacitação" (Murray e Lopez, 1996). O investimento na área de psiquiatria no Brasil é, portanto, crucial ante a prevalência, a morbidade e o impacto socioeconômico causados pelas doenças mentais.

\section{Referências}

Almeida-Filho N, Mari JJ, Coutinho E et al. Brazilian multicentric study of psychiatric morbidity. Methodological features and prevalence estimates. Br J Psychiatry, 171: 524-9, 1997.

Almeida OP. Autoria de artigos científicos: o que fazem os tais autores? Revista ABP-APAL, 20: 113-6, 1998.

Bressan RA, Gerolin J, Mari JJ. The modest but growing Brazilian presence in psychiatric, psychobiological and mental health research: assessment of the 1998-2002 period. Braz J Med Biol Res, 5: 649-59, 2005

Bressan RA, Miguel EC, Mari JJ et al. Chegamos ao ISI! Rev Bras Psiquiatr, 27: 170-1, 2005

Castro CM. Há produção científica no Brasil? In: Schwartzman S, Castro CM. Pesquisa universitária em questão. UNICAMP, p. 190-224 Campinas, 1986.

Coordenadoria de Aperfeiçoamento de Pessoal de Nível Superior - CAPES (2004). Disponível em: <www.capes.gov.br>. Acesso em: abril 2006.

Elkis $\mathrm{H}$. Fatores de impacto de publicações psiquiátricas e produtividade científica. Rev Bras Psiquiatr, 21: 231-6, 1999.

Journal of Citation Report - JCR (2004). Disponivel em: <http://esi3. isiknowledge.com/rankdatapage.cgi>. Acesso Abril 2006.
Garfield E. SCI Journal Citation Reports: a bibliometric analysis of science journals in the ISI database. Philadelphia, PA: Institute for Scientific Information, 1995.

In-Cities. Countries: January, 2003. Disponivel em: <http://www.in-cites. com/countries/brazil.html>. Acesso em: abril 2006.

In-Cities. Scy-Bites What's new in research: July 11, 2005. Disponivel em: <http://www.in-cites.com/research/2005/july_11_2005-1.html>. Acesso em abril 2006.

International Committee of Medical Journal Editors. Uniform Requirements for Manuscripts Submitted to Biomedical Journals. Annals of Internal Medicine, 126: 36-47, 1997.

Miranda DB, Pereira, MNF. O periódico científico como veículo de comunicação: uma revisão da literatura. Ci Inf, 25: 375-82, 1996.

Moed HF. The impact factors debate: the ISI's uses and limits. Nature, 415: 731-2, 2002

Murray CJL, Lopez AD. Evidence-based health policy - lessons from the global burden of disease. Science, 274: 740-3, 1996.

Shapiro D, Wenger N, Shapiro M. The contribution of authors to multiauthored biomedical research papers. JAMA, 271: 438-42, 1994. 